\title{
EVALUATION MODEL OF HUMANISTIC SYSTEMS BY FUZZY MULTIPLE INTEGRAL
}

\section{AND FUZZY CORRELATION}

\author{
Eiichiro Tazaki, Associate Professor \\ and Michio Amagasa, Research Associate \\ Faculty of Science and Technology \\ Science University of Tokyo \\ Noda City, Chiba 278, Japan
}

\section{Introduction}

The concept of fuzzy sets defined by Zadeh ${ }^{1}$ gives an important mathematical clue for an approach to studies of systems with no sharp boundaries. Lately, the fuzzy integ$\mathrm{ral}^{2}{ }^{2}$ based on the fuzzy set theory has been proposed and applied to the measurement of fuzzy objects, especially, pattern recognitions.

We consider the fuzzy integral is also available to be applied to the evaluation of fuzzy systems, because the fuzzy measure in the fuzzy integral will be regarded as a preference measure of evaluation and the fuzzy integral will represent a conflicting process between the preference measure and the evaluated object. When we consider the evaluation problem of complex systems composed of several subsystems, especially humanistic type ones, it is very difficult to set up the utility mode1 of overall system. Because, in general, the human preference has a hierarchical, strongly nonlinear and qualitative property. For such a case, we propose an utility model of evaluation by applying a fuzzy multiple integral. In this model the preference measure for the overall system is given by a composition of the subsystems' measure.

Applying the proposed method to a practical cases, the preference measure has to be determined by experiment. To realize such a process, we introduce a fuzzy distribution function and give an algorithm to calculate the measure. Further, we introduce a fuzzy correlation among subsystems in order that the fuzzy measure will be identified effectively by experimental data.

The proposed method is successfully applied to the subjective evaluation of a class of figures' largeness.

\section{Evaluation of complex systems by fuzzy multiple integral}

At first, we shall consider an evaluation of simple system composed of one subsystem as a preliminary for the evaluation of complex system.

The proposed model of evaluation is based on the fuzzy integral, where the fuzzy measure $g$ is considered as a measuring scale of preference, The fuzzy measure $g$ is given on a family of subsets $2^{x}$ with respect to $X$ as follows:

$$
\begin{aligned}
& 0 \leq \mathrm{g}\left(X^{\prime}\right) \leq 1, X^{\prime} \varepsilon 2^{X} \\
& X^{\prime} \subset X^{\prime \prime} \subset X \rightarrow g\left(X^{\prime}\right) \leq g\left(X^{\prime \prime}\right)
\end{aligned}
$$


Now, let $h(x)$ be a membership function with respect to the object of evaluation. Then the fuzzy integral is defined by

$$
f h(x) \circ g(\cdot)=V_{i=1}^{N}\left[h\left(x_{i}\right) \Lambda g\left(x_{i}\right)\right]
$$

where $x=\left\{x_{1}, x_{2}, \ldots, x_{N}\right\}, x_{i}=\left\{x_{1}, x_{2}, \ldots, x_{i}\right\}$.

The fuzzy integral will represent a conflicting process between the preference measure and the evaluated object. The fuzzy measure $\mathrm{g}$ is called, hereafter, the preference measure of evaluation. Therefore, if the fuzzy measure $g\left(X_{i}\right)$ is given for any subset of $2^{X}$, the fuzzy integral (3) can be calculated. In order to simplify the successive discussion, we assume that the rule for the generation of subsets with respect to the measure is defined as follows:

$$
g_{\lambda}\left(X^{\prime} U X^{\prime \prime}\right)=g_{\lambda}\left(X^{\prime}\right)+g_{\lambda}\left(X^{\prime \prime}\right)+\lambda g_{\lambda}\left(X^{\prime}\right) \cdot g_{\lambda}\left(X^{\prime \prime}\right) \quad-1<\lambda<\infty
$$

On the basis of the above mentioned results, we consider the evaluation of complex system $Z$ composed of two subsystems $X$ and $Y$. When the fuzzy measure for the subsystems $X$ and $Y$ are expressed by $g_{X}$ and $g_{Y}$, respectively, we will consider a product measure $g_{\lambda}$ in the product space $X \times Y$. Let $H$ be a family of subsets in $Z$, then $H$ is expressed the following form,

$$
\mathrm{H}=\mathrm{U}_{\mathbf{i}=1}^{\mathrm{N}}\left[\mathrm{X}_{\mathbf{i}} \times \mathrm{Y}_{\mathbf{i}}\right]
$$

where $\left\{x_{i}\right\},\left\{y_{i}\right\}$ are monotone increasing sequences formed by $x_{i}=\left\{x_{1}, x_{2}, \ldots, x_{i}\right\}$, $Y_{i}=\left\{y_{1}, y_{2}, \cdots-, y_{i}\right\}$, respectively.

The product measure $g_{\lambda}$ with respect to $\mathrm{H}$ is described as follows:

$$
g_{\lambda}(H)=V_{i=1}^{N}\left[g_{X}\left(X_{i}\right) \Lambda g_{Y}\left(Y_{i}\right)\right]
$$

Hence, the double integral with respect to the two variables function $h(z)=h(x, y)$ is defined as follows:

$$
\begin{aligned}
& v_{j=1}^{N}\left[V_{i=1}^{N}\left\{h\left(x_{i}, y_{j}\right) \Lambda g_{X}\left(x_{i}\right)\right\} \Lambda_{Y}\left(Y_{j}\right)\right] \\
& \left(=V\left[V\left\{h\left(x_{i}, y_{j}\right) \wedge g_{Y}\left(x_{j}\right)\right\} \Lambda g_{X}\left(x_{i}\right)\right]\right), \text { where } 0 \leq h \leq 1 .
\end{aligned}
$$

The equality in (7) is held when $h(z)$ is fuzzy measurable with respect to the monotone family $H C Z$. Thus, if the evaluation of overall system is given by the fuzzy double integral and if we apply such a method to the practical problems, the preference measure has to be identified. In order to realize the process, it is necessary to obtain the fuzzy distribution function and the fuzzy frequency function of each subsystem, respectively, given by the experiment.

Here we call the function $\mathrm{F}$ defined by the following equation the fuzzy distribution function.

$$
F\left(z_{i}\right)=V_{i=1}^{N}\left[F_{X}\left(X_{i}\right) \wedge F_{Y}\left(Y_{i}\right)\right]
$$

The function $F_{X}$ and $F_{Y}$ are, respectively, defined on the monotone sequence sets $\left\{X_{i}\right\}$ and $\left\{\mathrm{Y}_{i}\right\}$, and these functions have the following several properties.

$$
\begin{aligned}
& 0 \leq F_{X}\left(X_{i}\right) \leq 1, i=1,2, \ldots, N \\
& F\left(X_{i}\right) \leq F_{X}\left(X_{K}\right), i \leq k \\
& F_{X}\left(X_{N}\right)=1
\end{aligned}
$$

where $x_{i}=\left\{x_{1}, x_{2}, \cdots, x_{i}\right\}$, and $y_{i}=\left\{y_{1}, y_{2}, \ldots . ., y_{N+1-i}\right\}$ $F_{Y}$ has also the same properties as $F_{X}$, but the inequality sign is converse of $(10)$, 
and then $F_{Y}\left(Y_{1}\right)=1$. Hence, if the fuzzy distribution function is determined in each subsystem, the distribution function of overall system can be determined.

On the other hand, when the rule (4) is applied to the functions $g_{1}$ and $g_{2}$, respectively , the fuzzy frequency functions can be expressed by

$$
\begin{aligned}
& g_{1}^{1}=F_{X}\left(X_{1}\right), \quad g_{1}^{2}=\left(F_{X}\left(X_{2}\right)-F_{X}\left(X_{1}\right)\right) /\left(1+\lambda_{X} F_{X}\left(X_{1}\right)\right), \\
& g_{2}^{1}=F_{Y}\left(Y_{1}\right), g_{1}^{2}=\left(F_{X}\left(X_{i}\right)-F_{X}\left(X_{i-1}\right)\right) /\left(1+\lambda_{X} F_{X}\left(X_{i-1}\right)\right) \\
& \left.-F_{Y}\left(Y_{1}\right)-F_{Y}\left(Y_{2}\right)\right) /\left(1+\lambda_{Y} F_{Y}\left(Y_{2}\right)\right), \\
&
\end{aligned}
$$

where $-1<\lambda_{X}, \lambda_{Y}<\infty$.

In consequence, we define $g=g_{1} \times g_{2}$ on the set $S(Z)=U_{i=1}^{N}\left(X_{i} \times Y_{i}\right)$ composed of the monotone sequences of subsets in the system $Z$, and we define (14) as the fuzzy product frequency function.

$$
g(S(z))=v_{i=1}^{N} g_{1}^{i} \Lambda g_{2}^{i}
$$

Therefore, if the fuzzy distribution function of each subsystem can be given by using the experimental data, the fuzzv distribution function and the fuzzy frequency function of overall system can be, respectively, determined by equations (8), (12), (13) and (14).

\section{Fuzzy correlation}

We introduce a fuzzy correlation among subsystems in order that the fuzzy measure will be identified effectively by experimental data. We define the fuzzy correlation $n_{X Y}$ between the subsystems $X$ and $Y$ as well as it in the sense of statistics.

$$
n_{X Y}=\zeta_{X Y} / \zeta_{X} \zeta_{Y}
$$

where $\zeta_{X Y}, \zeta_{X}^{2}$ and $\zeta_{Y}^{2}$ are the fuzzy covariance and the fuzzy variances, respectively, and are represented as follows:

$$
\begin{aligned}
& \zeta_{X}^{2}=v_{j=1}^{N}\left(X_{i}-\mu_{X}\right)^{2} g_{1}^{i} \Lambda g_{X}\left(X_{i}\right) \\
& \zeta_{Y}^{2}=v_{i=1}^{N}\left(Y_{j}-\mu_{Y}\right)^{2} g_{2}^{1} \Lambda g_{Y}\left(Y_{i}\right) \\
& \left.\zeta_{X Y}=v_{j=1}^{N}\left\{v_{i=1}^{N}\left(X_{i}-\mu_{X}\right)\left(Y_{j}-\mu_{Y}\right) g\left(X_{i}, Y_{j}\right) \Lambda g_{X}\left(X_{i}\right)\right) \Lambda g_{Y}\left(Y_{j}\right)\right\} \\
& \mu_{X}=v_{i=1}^{N} X_{i} g_{1}^{j} \Lambda g_{X}\left(X_{i}\right) \\
& \mu_{Y}=v_{i=1}^{N}{ }_{Y} g_{2}^{1} \Lambda g_{Y}\left(Y_{i}\right)
\end{aligned}
$$

The fuzzy correlation $n_{X Y}$ shows the degree of connection among subsystems $X$ and $Y$ in fuzzifical sense. The range of its value is $\left|\eta_{X Y}\right| \leq 1$. In particular, if $n_{X Y}$ equals to zero, the subsystem $X$ is sufficiently independent from $Y$. Therefore, both of the fuzzy distribution function and the fuzzy frequency function of overall system can be, respectively, formulated by the product of isolated ones with respect to the subsystems $X$ and $Y$. Then, the evaluation of overall system is given by the product of the respective fuzzy integral for the subsystems $X$ and $Y$.

\section{Numerical example}

We will consider the length and extent of figures as the object of evaluation. We synthesize a mathematical model of the subjective evaluation as is shown in Fig.1. The proposed method will be inspected by experiments in the following section. 


\subsection{Construction of subjective evaluation}

The model of subjective eva-

luation is shown in Fig.1.

The $p$ and $\widehat{p}$ are stimulus variables from the object of evaluation and are input variables to the external and internal mechanisms, respectively. The $I$ and $\tilde{\pi}$ convert $p$ and $\tilde{p}$ to the inner evaluation $x^{i}$ and $\tilde{x}$, respectively. That is

$$
\pi: p+x^{i} \quad \tilde{I}: \tilde{p} \rightarrow \tilde{x}
$$

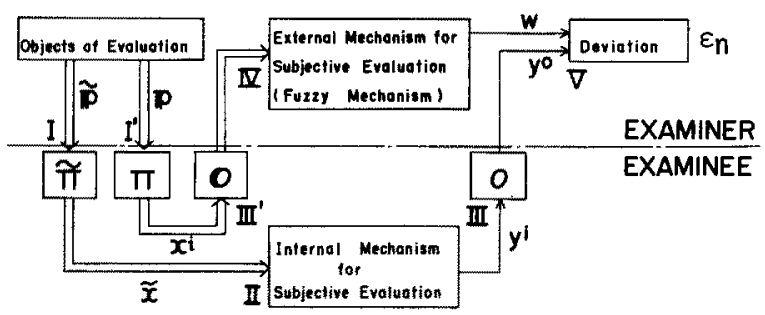

FIG. I Construction of Subjective Evoluation

The $y^{i}$ is an integrated inner evaluation which can be determined by means of the internal mechanism. The 0 and 0 are the interfaces so as to replace the inner evaluation with numerical value. And sense and ability of examinee with respect to number are also involved in it. The $w$ is an outer evaluation computed by the external mechanism. The $y^{0}$ is the outer evaluation obtained through the interface 0 .

The path $I \rightarrow \mathbb{I} \rightarrow \mathbb{I} \rightarrow \nabla$ shows the process of internal evaluation and the path $I \rightarrow \mathbb{I}^{\prime} \rightarrow \mathbb{N} \rightarrow \nabla$ shows the process of external evaluation. The $\varepsilon_{n}$ is a deviation of $w$ and $y^{0}$. In the external mechanism, we explain the fuzzy mechanism for the external evaluation in order to obtain the value of evaluation $w$,

The fuzzy mechanism is depicted in Fig.II.

\subsection{Computational algorithm}

In this section, we attempt to show a computational algorithm to calculate the fuzzy measure from experimental data. The algorithm will be easily obtained

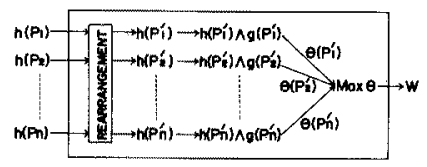

FIG. II FUZZY MECHANISM with reference to Fig.II as follows:

STEP 1 Formulate the membership function $h\left(p_{i}\right)$ for the objects of evaluation and rearrange the elements $h\left(p_{i}\right): i=1,2, \ldots, n$ according to the ordering of largeness as shown in Fig.II.

STEP 2 Calculate the fuzzy distribution and frequency functions according to (8), (12) , (13) and (14), respectively, from experimental data.

STEP 3 According to (4), determine the fuzzy preference measure using the fuzzy frequency function.

STEP 4 Compute the value of evaluation $w$ by means of the fuzzy mechanism.

STEP 5 Determine an optimal $\lambda$ so that the deviation $\varepsilon_{\mathrm{n}}$ is minimized.

\subsection{Formulation of the membership function with respect to shape and color}

We consider shapes and colors as attributes standing for length and extent of figures. The attribute of shape consists of the ratio of length and breadth, the ratio of the shortest and the longest length and the length of circuit etc., and the attribute of color consists of brightness, chroma and hue. We define the composed objective mem- 
bership functions of length and extent, respectively, subject to these attributes. Shape : $x=\left(x_{1}, x_{2}, x_{3}, x_{4}\right)=$ (the longest length, ratio of length and breadth, length of a circuit, ratio of the shortest length and the longest length)

Color : $Y=\left(Y_{1}, Y_{2}, Y_{3}\right)=($ brightness, chroma, hue $)$

$Z=(X, Y)=\left(Z_{1}, Z_{2}, Z_{3}, Z_{4}, Z_{5}, Z_{6}, Z_{7}\right)$

A) Membership function of length or extent with respect to shape and color

$$
h_{1}: z_{1} \times z_{2} \times-\cdots--\times z_{7} \rightarrow[0,1]
$$

The $h_{1}$ is the membership function for the length or extent of figure.

B) Membership function of length with respect to shape

$$
h_{2}: x_{1} \times x_{2} \rightarrow[0,1]
$$

C) Membership function of length with respect to color

$$
h_{3}: Y_{1} \times Y_{2} \times Y_{3} \rightarrow[0,1]
$$

We know that the color does not almost give an effect to the membership of 1ength in general.

D) Membership function of extent with respect to shape

$$
h_{4}: x_{3} \times x_{4} \rightarrow[0,1]
$$

E) Membership function of extent with respect to color

$$
h_{5}: Y_{1} \times Y_{2} \times Y_{3} \rightarrow[0,1]
$$

F) Membership function of length and extent with respect to shape and color

$$
h_{6}: h_{2} \times h_{3} \rightarrow[0,1] \quad h_{7}: h_{4} \times h_{5} \rightarrow[0,1]
$$

In practice, the proposed formulations of membership functions are, respectively, given by the followings:

A') $h_{1}=\sum_{i=1}^{K} M_{i} / K \quad$ The $M_{i}$ is a membership function corresponding to the fractionized part of shape.

B') $h_{2}=1 /(1+\alpha) \cdot\left(h_{i}+h_{j}\right)-0.05$, where $0.1<\alpha<1.0$ and $h_{i}$ and $h_{j}$ are the membership functions for the longest length of figure and the ratio of length and breadth, respectively, and $h_{i}=\log x, h_{j}=\log y$.

C') The membership function of length with respect to color is constant, that is $h_{3}=0.5$ D') $h_{4}=\alpha h_{i}+\beta h_{j},(\alpha+\beta=1)$, where $h_{i}$ is membership function of length of circuit and $h_{j}$ is the membership function of the ratio of the shortest length and the longest length. Further, $\alpha$ and $\beta$ are constant scalars, respectively.

E') $h_{5}=\alpha h_{i}+\beta h_{j}+\gamma h_{k}+c,(\alpha+\beta+\gamma=1)$, where $\alpha, \beta$ and $\gamma$ are constant scalars, respectively and $h_{i}, h_{j}$ and $h_{k}$ are the membership functions for brightness, chroma and hue, respectively.

Hence, the objective membership functions of length and extent can be composed by the above described membership functions. According to the above formulation, the objective memberships are obtained as shown in Tables 1,2,3 and 4. Further, on the basis of these results, the composed objective memberships with respect to shape and color are given by Tables 5 and 6 . 


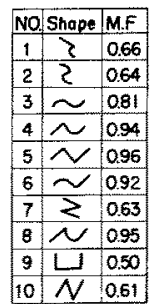

Tabie I. Woubership function of iength with respect to shape Basic Shape: NO.9

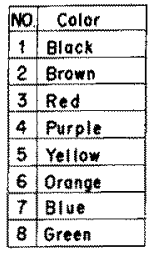

Toble 2. Mulbership function of length with respect to color Basic Color: NO.

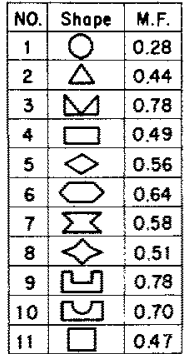

Table 3. Menbership function of extent with respect to shope. Basic Shope : NO. 11

\begin{tabular}{|c|l|c|}
\hline NO. & Color & M.F \\
\hline 1 & Red & 0.64 \\
\hline 2 & Orange & 0.69 \\
\hline 3 & Yellow Orange & 0.71 \\
\hline 4 & Yellow & 0.90 \\
\hline 5 & Yellow Green & 0.75 \\
\hline 6 & Green & 0.54 \\
\hline 7 & Green Blue & 0.52 \\
\hline 8 & Blue Green & 0.43 \\
\hline 9 & Olue & 0.46 \\
\hline 10 & Blue Purple & 0.27 \\
\hline 11 & Purple & 0.30 \\
\hline 12 & Red Purple & 0.31 \\
\hline
\end{tabular}

Table 4. Manberstip function of extent with respect to color Bosic Color: NO. 7
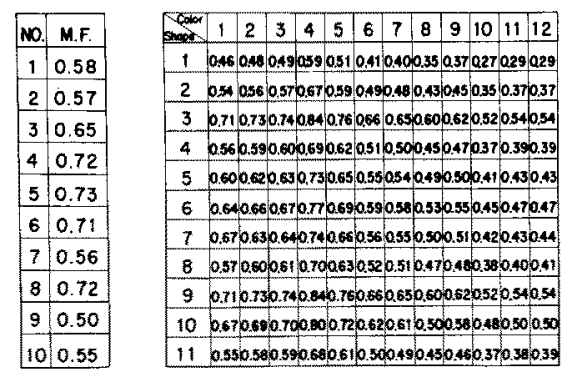

Table 5. Composed Table 6. Composed membership function of extent. membership function of

fength with respect to shope and calor.

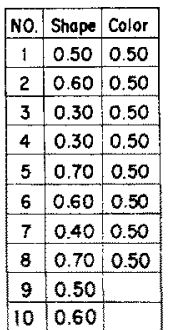

Table 7. Resulls of an experimen for length with respect to shape and color.

\begin{tabular}{|c|c|c|}
\hline NO & Shape & Color \\
\hline 1 & 0.40 & 0.50 \\
\hline 2 & 0.60 & 0.40 \\
\hline 3 & 0.70 & 0.50 \\
\hline 4 & 0.50 & 0.40 \\
\hline 5 & 0.60 & 0.40 \\
\hline 6 & 0.50 & 0.50 \\
\hline 7 & 0.60 & 0.50 \\
\hline 8 & 0.50 & 0.60 \\
\hline 9 & 0.60 & 0.70 \\
\hline 10 & 0.70 & 0.60 \\
\hline 11 & 0.50 & 0.70 \\
\hline 12 & & 0.70 \\
\hline
\end{tabular}

Tobie 8 . Resutts of on experiment for extent with respect to shope and color.

On the other hand, in order to identify the preference measure of evaluation, we had an experiment such that examinees evaluate the length and extent of figures. As the results of experiment, we have obtained the values of evaluation with respect to shape and color as shown in Tables 7 and 8 .

According to the computational algorithm described in section 4.2 , an optimal $\lambda$ for the preference measure is given by Table 9 .

In order to inspect an advantage of the proposed method, the evaluation of figures is executed by using the calculated measure. The comparison of computed results and experimental results is shown by Table 10. It shows that a good consistency is held

\begin{tabular}{|c|c|c|c|}
\hline & $W$ & $y^{0}$ & $\lambda$ \\
\hline Length (Shape) & 0.61 & 0.60 & -0.98 \\
\hline LengthiShape,Coky) & 0.57 & 0.60 & -0.20 \\
\hline Extent(Shape.Colot & 0.45 & 0.40 & 1.00 \\
\hline
\end{tabular}

Toble 9. Exomple of optimal $\lambda$ within the limit of this problem.

\section{Conclusion}

We have proposed a mathematical model of subjective evaluation on the basis of fuzzy integra1. In particular, we have developed the evaluating method of complex systems

$\lambda * \mid$
\begin{tabular}{|l|c|c|c|}
\hline Extent & $w$ & $y^{0}$ & $\epsilon_{\mathrm{n}}$ \\
\hline Figurel & 0.565 & 0.500 & 0.065 \\
\hline Figure2 & 0.505 & 0.600 & 0.095 \\
\hline
\end{tabular}

Table 10. Comparison of a computed resulf and an experimental result, composed of several subsystems by vintue of fuzzy multiple integral. 
Furthermore, for the sake of identifying the preference measure, that is fuzzy measure , effectively, we have introduced the fuzzy correlation.

In order to show how the proposed method works, an example of the subjective evaluation for a class of figures has been illustrated and the consistency of experimental values and computed values has been successfully obtained as shown in Table 10.

Applications to the marginal evaluation problem to meet specification will be a future extension of this approach.

\section{References}

1) L.A.Zadeh : Fuzzy Sets, Information and Control, 8, 338/353 (1965)

2) M.Sugeno: Fuzzy Measure and Fuzzy Integra1, Trans. SICE(Japan), 8, 218/226 (1972)

3) E.Tazaki and M.Amagasa : Evaluation of Complex Systems by Fuzzy Multiple Integral and Fuzzy Correlation, Paper presented to the 16-th Joint Annual Meeting of Automatic Control, Tokyo, Japan (1973) 